\title{
Reactivation of BKV and AdV infections during post-transplant immunosuppressive therapy
}

\author{
Adrian Jarzyński', Justyna Nowotnik', Anna Zagaja², Dariusz Pachnia', \\ Małgorzata Polz-Dacewicz ${ }^{1}$ \\ ${ }^{1}$ Department of Virology, Medical University of Lublin \\ ${ }^{2}$ Department of Ethics and Human Philosophy, Medical University of Lublin
}

Jarzyński A, Nowotnik J, Zagaja A, Pachnia D, Polz - Dacewicz M. Reactivation of BKV and AdV infections during post-transplant immunosuppressive therapy. J Pre-Clin Clin Res. 2013; 7(2): 93-97.

\begin{abstract}
Introduction. Viral infections are an important problem in transplantology. Infections in recipients may develop as a result of the original infection or reactivation of a latent infection. Many viruses have the ability to enter into a latent state without symptoms. The most common groups of viruses causing infections in patients after transplantations are herpersviruses, hepatotropic viruses, retroviruses, adenoviruses and poliomaviruses.

Objectives. The aim of the presented study is to analyze the incidence of infections caused by BKV and AdV in a group of patients after kidney and bone marrow transplant.

Material and methods. The study group consisted of 13 patients after bone marrow transplantation and a group of 10 patients after kidney transplantation. DNA was isolated from urine and serum and analysed with PCR auto-nested reaction. The amplification products were separated in agarose gel.

Results. A positive result for AdV was recorded in 7 patients after bone marrow transplant and 5 patients after renal transplant. BK virus was detected in the urine of 13 patients, and in the blood sample of 5 patients.

Discussion. Reactivation of a virus during immunosuppression is a frequent cause of graft rejection. In addition, in patients with impaired immune system, mixed infections with various types of viruses are becoming a serious problem. This study confirm that infections with BKV and AdV viruses are a major problem in the field of transplantation.

Conclusion. BKV and AdV are common causes of infections affecting patients after renal and bone marrow transplantation. BKV infections are often accompanied by human adenovirus infection. Adenovirus infections are a more common cause of infection in bone marrow transplant patients than in patients after renal transplantation.
\end{abstract}

\section{Key words}

Adenovirus, BK virus, immunosuppression, transplantation, bone marrow, kidney

\section{INTRODUCTION}

Opportunistic infections, which may lead to serious, irreversible dysfunctions, rejection of the transplanted organ, and in severe cases, even to the patient's death, are an important problem in transplantology. Immunosuppression inhibits one of the basic mechanisms of antiviral defence cytotoxic T. lymphocytes. This enhances viral proliferation, possible reactivation of latent infections and rapid spread of the disease. Infections in recipients may develop as a result of the original infection (during the first contact with the pathogen) or as an outcome of the reactivation of a latent infection. Many viruses have the ability to enter into a latent state without symptoms. However, under the influence of certain factors, most commonly immunity decrease, reactivation of the virus can appear. There are three basic stages of post-transplant infections: the early stage (up to one month after transplantation), which includes infections caused by the surgery itself; the intermediate period $(2-6$ months after transplantation), where primary infections with viruses such as CMV, HHV-6, EBV, AdV, BKV, bacteria, fungi and infusoria occur, and the late period (more than half a year after transplantation), where common infection for the recipients include those most common in the general

Address for correspondence: Adrian Jarzyński, Department of Virology, Medical University of Lublin, Chodźki 1, 20-093 Lublin, Poland e-mail: a.jarzynski87@gmail.com

Received: 01 August 2013; accepted: 04 February 2014 population, such as infections caused by the influenza virus, parainfluenza, RSV or urinary tract infection.

Most common post-transplant viral infections. The most common groups of viruses causing infections in patients after transplantations are herpersviruses, hepatotropic viruses, retroviruses, adenoviruses and poliomaviruses [1].

Adenovirus. The majority of the patients become infected by at least one serotype of AdV before turning fifteen. There exists a connection between the frequency of isolating various types of viruses and the patient's age (newborns type:1, 2,3, $5,6,7$; older children 3,7 ; adults: $3,4,7$, sometimes 14 ). In patients after transplantations the adenoviral infection rate varied from $5 \%-47 \%$ [2].

Polyomavirus. the most common of these viruses is the BKV virus. Approximately $60-90 \%$ of the world population is infected with it and its reactivation occurs in $23-57 \%$ of renal recipients. $1-10 \%$ of the recipients suffer from nephropathy leading to irreversible damage to the grafted kidney [3].

Characteristics of AdV. AdV belongs to the genus Mastadenovius, of the family Adenoviridae. It is a type of virus that infects only mammals, unlike the other type of virus from this family, i.e Aviadenovirus which infects bird.

Currently, there are 52 known serotypes of AdV, which have been divided into 6 subgenera, labeled A - F, and contained in each subgroup are several types of virus. The criterion for this division is the sequence of nucleotides in the DNA [4]. 
Adenovirus virion is composed of a single molecule of double-stranded DNA constituting $12-17 \%$ of the virus. The capsid is icosahedral in shape and composed of 252 capsomeres ( 240 hexons and 12 pentons). Each penton has a projecting protein fibre with a terminal knob 10-37 nm in length, depending on the type of virus. Virions do not have a lipid shell, and are approximately $80 \mathrm{~nm}$ in diameter. Virions of adenoviruses exhibit stability in terms of $\mathrm{pH}$ 6-9). Fat solvents and small chlorine concentration do not influence the virus. Adenoviruses retain their viability in the temperature between $-25^{\circ}$ and $-70^{\circ}$ for about 2 months; however, after being heated to $56^{\circ}$ for 10 minutes they become inactivated [5].

Adenovirus infections are frequent and constitute up to approximately $13 \%$ of all human viral infections [6]. Adenoviruses are one of the most frequent causes of diarrheas in paediatric patients and affect mainly infants and children up to 2 years of age. In older age, the human body produces antibodies against the so-called intestinal adenoviruses, $\mathrm{AdV} 40$ and 41 [7]. In adults, gastrointestinal infections are usually mild and self-limiting. In infants, the infection is slightly more severe than in older children, also concomitant infections of the throat and the respiratory tract are more common in infants and cover $80 \%$ of cases, whereas in older children $-31 \%$. Another condition caused by the AdV is tonsillitis.

The only criterion allowing for the differentiation between viral and bacterial tonsillitis is age. Infections caused by AdV affect children below 3 years of age, while bacterial infections occur between the ages of 5 and 17 [6]. Among the most common ophthalmic infections are conjunctivitis and keratitis. In the case of keratitis, after few days from infection, point-changes appear on the eye's surface; however, they do not require treatment and disappear within couple of weeks [8]. Adnoviruses, mainly type 11, are also responsible for haemorrhagic cystitis in children. This disease is more common in boys, and its symptoms include bloody urine, frequent urination, fever, burning pain in the abdomen and painful urinary urgency [9]. Neurological complications caused by adenoviruses are rare, but very dangerous due to the high risk of death. The disease most commonly affects newborns and immunocompromised people.

Characteristic of BKV. The $\mathrm{BK}$ virus belongs to the Polyomaviridae family and the Polyomavirus genus. Currently, there are 5 known human polyomaviruses: BKV, JCV, WUV, KIV and MCV. There are 4 subtypes of BKV, labeled I - IV, including 4 sub-groups within the subtype I (a, b1, b2, c) [10].

The genome of the BK virus consists of a single-stranded, circular DNA molecule with a length of 5'153 bp. The genetic material is enclosed in an icosahedral capsid, approximately $45 \mathrm{~nm}$ in diameter. The capsid of the polyomaviruses is composed of 3 proteins: VP1, VP2 and VP3, which are made up of 72 capsomeres, grouped into 12 pentamers and 60 heksamers [10].

Polyomavirus infections are very common although it is estimated that $70-90 \%$ of the adult population has antibodies against $\mathrm{BK}$ and JC virus. These infections, usually occurs in early childhood and are asymptomatic. They do not pose a threat in immunocompetent individuals; however, in patients with reduced immunity, particularly after kidney and bone marrow transplantation, they are the cause of serious infections [11]. Modes of BKV transmission have not been fully recognized, although it is believed that the viruses are transmitted through respiratory droplets, the faecal-oral route, after exposure to infected blood, including organ transplants, and through the placenta [12]. As a result of childhood infections, the viruses penetrate the tissue where they undergo latency. The main location of the BKV virus latency is considered to be the urinary tract. After application of immunosuppressive therapy, which lowers the body's resistance, BK virus and other polyomavirus, are reactivated. They may cause respiratory infections (mainly KIV and WUV), interstitial nephritis in transplant recipients (BKV), haemorrhagic cystitis after bone marrow or kidney transplantation, and progressive multifocal leukoencephalopathy (JCV) [10].

Aim of the study. The aim of the study was to analyze the incidence of infections caused by BKV and AdV in a group of patients after kidney and bone marrow transplant. The relationship between $\mathrm{BKV}$ infection and the incidence of AdV infection was also analyzed.

\section{MATERIALS AND METHOD}

Characteristic of the population and the research material. The study group consisted of 13 patients after bone marrow transplantation (11 children and 2 adults) treated in the Independent Public Clinical Hospital No. 4. in Lublin (PSK4), and in the Children's Clinical Hospital (DSK), both in Lublin, south-eastern Poland, as well as a group of 10 patients after kidney transplantation from PSK4.

The research material consisted of 23 urine and serum samples obtained from 23 patients infected with the BK virus. The material was stored in sterile test tubes and frozen at $-23^{\circ} \mathrm{C}$ until analysis.

Isolation of viral DNA from urine sample. Conducted using the Viral DNA Kit (QIAGEN), in accordance with the standard procedure provided by the manufacturer.

Isolation of viral DNA from serum. Conducted using DNA Blood Mini-Kit (QIAGEN), in accordance with the standard procedure provided by the manufacturer.

PCR auto-nested. A method for increasing sensitivity based on performing 2 successive reactions, out and in. The products obtained in the 'out' reaction are the matrix for the 'in' reactions. $1 \mu \mathrm{l}$ of the product is transferred from the 'out' reaction to the 'in' reaction (the volume designated for the isolate should be refilled with water, the other ingredients and the profile of the reaction without change).

The out PCR reaction BKV/AdV. The volume for one sample equaled $20 \mu \mathrm{l}$ (water for PCR - $7 \mu \mathrm{l}$; 10x bufor PCR - $2 \mu \mathrm{l}$; Bufor Q - $4 \mu \mathrm{l}$; $\mathrm{MgCl}_{2-} 0.6 \mu \mathrm{l}$; dNTPs - $0.4 \mu \mathrm{l}$; primer PEP 1 / AZH 1 - $0.5 \mu \mathrm{l}$; primer PEP 2 / AZH 4R - $0.5 \mu$; Taq Hot Start polimerase $-0.1 \mu \mathrm{l})$. After stirring it was divided into $15 \mu \mathrm{l}$ and $5 \mu \mathrm{l}$ of DNA isolate was added respectively to each. Primer sequence for BKV: PEP 1 - 5' AGT CTT TAG GGT CTT CTA CC 3'; PEP 2 - 5' GGT GCC AAC CTA TGG AAC AG 3'. Primer sequence for AdV: AZH 1 - 5' GCC GAG AAG GGC GTG CGC AGG TA 3’; AZH 4R - 5’ ATG ACT TTT 
GAG GTG GAT CCC ATG GA 3’. Amplification was conducted in a thermocycler in the conditions described in Table 1. Size of the PCR products of the 'out' reaction: BKV176 bp, AdV-150 bp.

Tables 1. BKV/AdV PCR thermocycler conditions.

\begin{tabular}{cc}
\hline Temperature & Time (amount of cycles) \\
\hline $95^{\circ} \mathrm{C}$ & 15 minutes \\
\hline $94^{\circ} \mathrm{C}$ & 1 minute $(35$ cycles $)$ \\
\hline $55^{\circ} \mathrm{C}$ & 1 minute $(35$ cycles $)$ \\
\hline $72^{\circ} \mathrm{C}$ & 1 minute $(35$ cycles $)$ \\
\hline $72^{\circ} \mathrm{C}$ & 5 minuts \\
\hline
\end{tabular}

Restriction. To distinguish BK from JC virus it is necessary to conduct a restriction enzyme digestion with FokI BKVspecific, as a result of which, after electrophoresis under an UV lamp, it will be possible to observe 2 bands for BKV and 1 for JCV.

Mix $10 \mu \mathrm{l}$ of the product of reaction, $16 \mu \mathrm{l}$ water, $1-2 \mu \mathrm{l}$ of the FokI enzyme and $2 \mu \mathrm{l}$ of $10 \mathrm{x}$ buffer Tango. Incubate at $55^{\circ} \mathrm{C}$ for 15 hours; then at $80^{\circ} \mathrm{C}$ for 20 minutes to inactivate the enzyme and detect in agarose gel.

Detection. Amplification products are separated in a $2 \%$ agarose gel at $96 \mathrm{~V}$ for about 20-30 minutes. In the case of BKV, 2 DNA bands with sizes of $120 \mathrm{bp}$ and $56 \mathrm{bp}$, and for JCV 1 band with a size of 176 bp is observed under UV light.

\section{RESULTS}

Occurrence of AdV in patients after bone marrow transplant. The study group consisted of 13 participants. A positive result for AdV was recorded in 7 of them (53.8\%) and a negative in 6 (46.2\%) (Fig. 1).

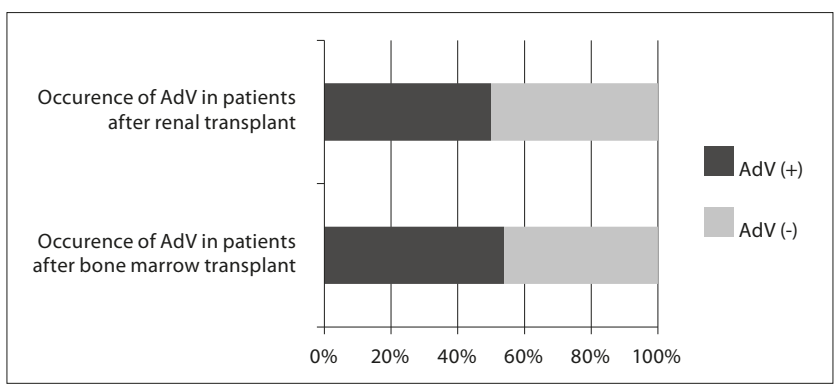

Figure 1. Percentage analysis of AdV occurrence in patients after bone marrow and renal transplant

Occurrence of AdV in patients after a renal transplant. The study group consisted of 10 participants. A positive result for $\mathrm{AdV}$ was recorded in half of them (50\%) (Fig. 1).

Analysis of the presence of BKV and AdV in the urine and blood samples of patients after bone marrow transplantation. The presence of adenovirus was found in the urine of 7 patients (53.8\%) and in the blood of one patient $(7.7 \%)$. BK virus was detected in the urine of 13 patients (100\%), and in the blood sample of 5 patients (38.5\%) (Fig. 2, Tab. 2).
Table 2. Results of BKV/AdV DNA in patients after bone marrow transplant

\begin{tabular}{lll}
\hline BKV/AdV DNA & \multicolumn{2}{c}{ Number of patients } \\
\cline { 2 - 3 } & \multicolumn{1}{c}{ BKV } & AdV \\
\hline Urine + / blood + & $5(38.5 \%)$ & $1(7.7 \%)$ \\
\hline Urine + / blood - & $8(61.5 \%)$ & $6(46.2 \%)$ \\
\hline Urine - / blood + & $0(0 \%)$ & $0(0 \%)$ \\
\hline Urine - / blood - & $0(0 \%)$ & $6(46.2 \%)$ \\
\hline
\end{tabular}

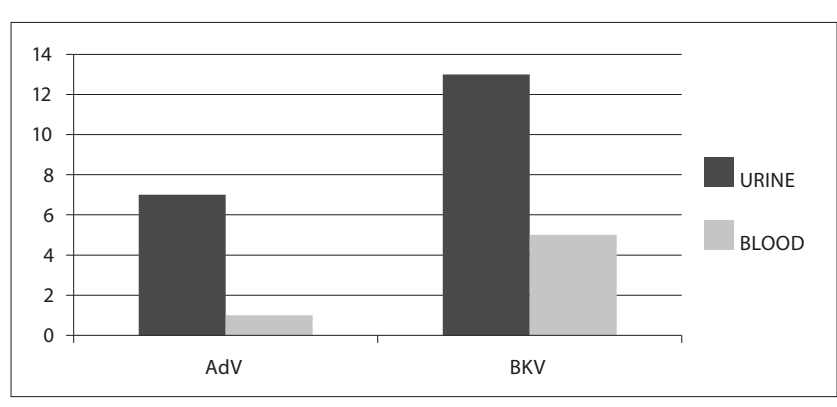

Figure 2. Analysis of viruria and viremia levels caused by AdV and BKV in patients after bone marrow transplant

Analysis of BKV and AdV presence in urine and blood samples in patients after renal transplant. The presence of adenoviruses was found in the urine of 5 patients (50\%) and in blood samples of $2(20 \%)$. BKV was found in the urine of all of the patients (100\%) and in the blood of 1 (10\%) (Fig. 3, Tab. 3).

Table 3. Results of BKV/AdV DNA in patients after renal transplant.

\begin{tabular}{lll}
\hline \multirow{2}{*}{ BKV/AdV DNA } & \multicolumn{2}{c}{ Number of patients } \\
\cline { 2 - 3 } & BKV & AdV \\
\hline Urine + / blood + & $1(10 \%)$ & $2(20 \%)$ \\
\hline Urine + / blood - & $9(90 \%)$ & $3(30 \%)$ \\
\hline Urine - / blood + & $0(0 \%)$ & $0(0 \%)$ \\
\hline Urine - / blood - & $0(0 \%)$ & $5(50 \%)$ \\
\hline
\end{tabular}

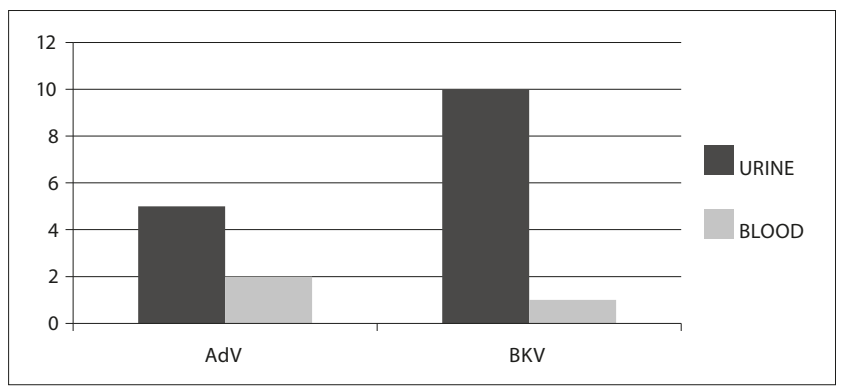

Figure 3. Analysis of viruria and viremia levels caused by AdV and BKV in patients after a renal transplant

The course of AdV and BKV infections in patients after bone marrow transplant. $\mathrm{BKV}$ was present in the urine throughout the period of testing in all the patients. In 5 of the 6 patients, BKV infection was accompanied by adenovirus infection, manifested by the presence of AdV DNA in urine or blood. 1 patient had a full-blown infection where BKV and $\mathrm{AdV}$ were present in both urine and blood samples (Tab. 4). 
Table 4. Course of $A d V$ and BKV infection in patients after bone marrow transplant.

\begin{tabular}{|c|c|c|c|c|c|}
\hline & \multirow[t]{2}{*}{ Date of the study } & \multicolumn{2}{|c|}{ BKV } & \multicolumn{2}{|c|}{ AdV } \\
\hline & & BLOOD & URINE & BLOOD & URINE \\
\hline \multirow[t]{5}{*}{ Patient 1} & 21.04.2009 & - & + & - & - \\
\hline & 28.04.2009 & + & + & - & + \\
\hline & 05.05.2009 & + & + & - & - \\
\hline & 12.05 .2009 & - & + & - & - \\
\hline & 19.05.2009 & - & + & - & - \\
\hline \multirow[t]{5}{*}{ Patient 2} & 04.06 .2009 & - & + & + & - \\
\hline & 12.06 .2009 & - & + & - & - \\
\hline & 17.06.2009 & + & + & - & - \\
\hline & 23.06 .2009 & + & + & - & - \\
\hline & 17.07.2009 & + & + & + & - \\
\hline \multirow[t]{2}{*}{ Patient 3} & 22.10 .2009 & + & + & + & + \\
\hline & 27.10 .2009 & - & + & - & - \\
\hline \multirow[t]{2}{*}{ Patient 4} & 19.11.2010 & - & + & - & - \\
\hline & 19.12.2010 & - & + & - & - \\
\hline \multirow[t]{2}{*}{ Patient 5} & 21.12 .2010 & - & + & - & - \\
\hline & 30.12 .2010 & - & + & - & + \\
\hline \multirow[t]{4}{*}{ Patient 6} & 20.01 .2011 & + & + & - & - \\
\hline & 25.01 .2011 & + & + & - & - \\
\hline & 02.03 .2011 & - & + & - & + \\
\hline & 16.03 .2011 & - & + & - & + \\
\hline
\end{tabular}

\section{DISCUSSION}

Because of the continuous development in the field of transplantation, both that of solid organs as well as hematopoietic cells, and the immunosuppression associated with it, viral infections may occur more frequently and with more severe symptoms. In addition, in patients with impaired immune system, mixed infections with various types of viruses are becoming a serious problem. Reactivation of a virus during immunosuppression is a frequent cause of graft rejection. That is why an effective viral diagnosis and undertaking quick treatment of such infections is crucial [6].

The incidence of adenovirus infections in transplant patients has increased significantly in recent years. This may be due to several reasons: greater awareness of the pathogenicity of the virus, intensified immunosuppressive therapy, more sensitive diagnostic methods and systematic control. AdV infections ranges from 5\% - 47\%, depending on the age of the patient, type of diagnostic method, and the analyzed sample [2]. Mortality among patients ranges from $10 \%$ to as much as $80 \%$. Children fall sick more often than adults because they are more susceptible to primary infection or reactivation of latent infections. A larger amount of AdV DNA was detected in the tonsils of children under 9 years of age than in older ones. It is believed that the more frequent reactivation of adenoviral infection is caused by abandoning the once standard practice of tonsillectomy in young children [13]. Higher mortality due to AdV infection has been reported in patients after transplantation from unrelated donors, patients with acute lymphocytopenia after antilymphocyte globulin therapy and in cases of graft-versus-host disease [2].

Clinical symptoms in patients who are infected with adenovirus include infections of the upper and lower respiratory tract, interstitial pneumonia, hepatitis, diseases of the genitourinary system, such as haemorrhagic cystitis or inflammation of the kidneys, and digestive system diseases, such as haemorrhagic colitis. AdV in patients after bone marrow transplantation is typically detected within 100 days of transplantation [2]. The disease can be either located within a single organ or disseminated throughout the body. Regular blood tests are now widely used in AdV infection prevention in patients after transplantation, particularly in children. The virus can be detected in the blood from $2-3$ weeks before the development of clinical symptoms, which allows for rapid implementation of treatment [14]. Various serotypes of adenoviruses are isolated from patients, most often these are viruses belonging to the A, B and C subgenus. Patients with mixed infections exhibit a longer period of virus excretion than patients infected with only 1 serotype [2].

Patients after transplantation, especially bone marrow transplantation, are particularly vulnerable to infections of the respiratory tract which can even prove fatal. Adenovirus infections in children are detected, on average, in less than 30 days after transplantation, and in adults even less than 90 days, and detected in approximately $14 \%$ of patients [15]. Risk factors conductive to adenovirus infection include: graft-versus-host disease, herpes virus infection and isolating adenoviruses from various clinical specimens. Mortality resulting from $\mathrm{AdV}$ infection reaches up to $50 \%$, despite the use of antiviral therapy [16].

Adenovirus infections are a frequent cause of diseases in renal transplant recipients. In most patients, the infection is manifested by haemorrhagic cystitis present with fever, haematuria and soreness during micturition. In a study conducted by Watcharananan et al., nearly $2 / 3$ of patients showed severe dysfunction of the transplanted organ. Average time of onset of the infection is 5 weeks after transplantation [17].

According to various studies, the estimated frequency of AdV infection in patients after HSCT ranges from $3 \%-47 \%$ [18]. In the study conducted by Bil-Lula et al., adenoviral infection concerned up to $44.8 \%$ of patients after transplantation of hematopoietic stem cells.

AdV DNA was detected in $55.8 \%$ of urine samples and in $1.9 \%$ of serum samples, and $28.8 \%$ of patients showed disseminated infection, where the presence of AdV was detected in both urine and blood. The presented study also demonstrated that $\mathrm{AdV}$ infection is often accompanied by the BKV infection. The authors of the presented study also argue that $\mathrm{BKV}$ and $\mathrm{AdV}$ infection occur most commonly as co-infections [18].

$\mathrm{BK}$ virus is a major etiologic factor in interstitial nephritis in patients who have undergone renal transplant [10]. The reactivation of the $\mathrm{BK}$ virus affects $10-60 \%$ of patients after renal transplantation. As demonstrated in the literature, nephropathy associated with BKV reactivation occurs in $8 \%$ of recipients, causing transplant rejection in up to $50 \%$ of cases [19].

BK virus reactivation is an important cause for developing various symptoms in patients after renal transplantation. This seemingly insignificant infection during immunosuppression is a major contributory factor to the loss of the transplanted organ. The symptoms of infection can be detected in more than $80 \%$ of adults. Anti-BKV antibodies are detected in $73 \%$ of renal allograft recipients. It has not been established whether the infections caused by the $\mathrm{BK}$ virus in transplant recipients result from the reactivation of persistent infection, or whether the recipients are infected with the virus derived from the donor [20].

The main locations where the virus becomes latent, are renal tubular epithelial cells and the urinary tract, as well as lymphoid cells or brain tissue. BKV reactivation occurs in people with 
weakened immune systems, resulting from immunosuppressive therapy after transplantation. Other factors contributing to infection may include: pregnancy, chemotherapy, HIV infection, uncontrolled diabetes, the use of cytotoxic drugs, the presence of anti-BKV antibodies in the donor organ, mismatch of the donor's and recipient's HLA tissue types, and the age and genderof the patient (older, male) [21].

Clinical manifestations of BK virus infection usually include interstitial nephritis, ureteral stenosis and haemorrhagic cystitis. BKV can cause graft dysfunction, it is estimated that the BK nephropathy occurs in $2-5 \%$ of recipients, and is the cause of organ rejection in $45 \%$ of cases. The disease may begin within 4 months after kidney transplantation and continue until graft failure, with an average diagnosis time of 9.5 months [21]. At its earliest, nephropathy was diagnosed as early as the 6th day, the latest 6 years after transplantation [22].

In the past few years, in renal transplant patients, the incidence of BKV caused nephropathy has increased. This is probably caused by the increase in the amount of transplantation procedures, as well as more effective diagnostic methods. Until recently, BKV nephropathy was misdiagnosed as acute transplant rejection, which resulted in improper treatment, intensification of the disease, and ultimately graft loss. Interstitial nephritis can continue for months without giving visible symptoms. The final diagnosis is confirmed by a renal biopsy in order to detect morphological changes caused by viral replication. Sometimes, the biopsy can give a false negative result due to the focal nature of the disease. The development of the disease can be observed using the initial detection of the virus in urine, then blood, and finally in the kidneys of the patient. BK nephropathy is detected in approximately $8 \%$ of patients after kidney transplantation, resulting in graft loss in $50 \%$ of cases within 2-3 years [19]. In a study conducted by Sung et al., viraemia was detected in $18.8 \%(12 / 64)$ and viruria in $28.1 \%(18 / 64)$ of patients after renal transplant [23].

Haemorrhagic cystitis (HC) is a very common complication in renal transplant recipients and allogeneic haematopoietic stem cell transplantation (HSCT). The disease occurs in $5-40 \%$ of patients after HSCT [24]. However, in $50-100 \%$ of recipients, $\mathrm{BK}$ viruria is detected; thus, the mere presence of the virus in the urine is insufficient to cause the disease [25]. Risk factors favouring the development of haemorrhagic cystitis include: acute form of graft-versus-host disease, a specific mutation of $\mathrm{BKV}$, the type of immunosuppressive drugs used, and BK virus subtype, and a graft obtained from an unrelated donor. Haemorrhagic cystitis in patients after haematopoietic cell transplantation usually develops late in the post-translational period. However, $\mathrm{HC}$ can develop even a few days after transplantation. In the studies conducted by Erard et al., in 33\% of recipients of allogeneic haematopoietic cell transplantation in whom BKV viraemia was detected, the development of $\mathrm{HC}$ occurred in $43 \%$ of the cases, an average of 9 days after transplantation [10].

In the presented study, BK virus infection occurred in $100 \%$ of the cases, whereas AdV infection concerned $52.2 \%$ of all patients. These studies confirm that infections with these viruses are a major problem in the field of transplantation.

\section{CONCLUSIONS}

$\mathrm{BKV}$ and $\mathrm{AdV}$ are common causes of infections affecting patients after renal and bone marrow transplantation. BKV infections are often accompanied by human adenovirus infection. Adenovirus infections are a more common cause of infection in bone marrow transplant patients than in patients after renal transplantation. BKV DNA and AdV are more frequently isolated from urine samples than blood samples.

\section{REFERENCES}

1.Skrzypek A. Infectious complications after kidney transplantation. Przegląd urologiczny 2007; 8: 41.

2. Echavarria M. Adenoviruses in immunocompromised host. Clin Microbiol Rev. 2008; 21: 705-715.

3. $\mathrm{Hu} \mathrm{JH}$, Zhao H, Huang YP, Zhang X. Oportunistic posttransplantation virus infections in renal transplantant recipients. Transplant Proceed. 2011; 43: 3715-3719.

4. Collier L, Oxford J. Human Virology. 3th Ed. PZWL. Warsaw, 1996. p.102-146.

5. Kańtoch M. Medical Virology. 2nd Ed. PZWL. Warsaw, 1998. p.68-187.

6. Dzieciątkowski T, Rola A, Midak-Siewirska A. Adenoviral infection of humans. Post Mikrobiol. 2008; 47: 15-22.

7. Kotloff KL, Losansky GA, Morris JG Jr, et al. Enteric adenovirus infectionand childhood diarrhea: an epidemiologic study in three clinical setting. Pediatrics. 1989; 84: 219-225

8. Butt AL, Chodosh J. Adenoviral keratoconjuncitivitis in a tretiary care eye clinic. Cronea. 2006; 25: 199-201.

9. Shindo K, Kitayama T, Ura T, et al. Acute hemorrhagic cystitis caused by adenovirus type 11 after transplantation. Urologia Internationalis. 1986; 41: 152-155.

10. Ryanans S, Dzieciątkowski T, Młynarczyk G. Human polyoma viruses infection in immunosuppressed patients. Post Mikrobiol. 2011; 50: 191-199.

11. Boothpur R, Brennan DC. Human polyoma viruses and disease with emphasis on clinical BK and JC. J Clin Virol. 2010; 47: 306-312.

12. Jeffers LK, Madden V, Webster-Cyriaque J. BK virus has tropism for human salilary gland cells in vitro: implications for transmission. Virology. 2009; 394: 183-193.

13. Garnett CT, Pao CI, Gooding LR. Detection and quantitation of subgroup C adenovirus DNA in human tissue samples by real-time PCR. Methods Mol Med. 2007; 130: 193-204.

14.Lion T, Baurngartiner R, Watzinger F, et al. Molecular monitoring of adenovirus in peripheral blood after allogeneic bone marrow transplantation permits early diagnosis of disseminated disease. Blood. 1003; 60: 133-140.

15. Leen AM, Bollard CM, Myers GD, Rooney CM. Adenoviral infections in hematopoietic stem cell transplantation. Biol Blood Marrow Transplant. 2006; 12: 243-251

16. Flomenberg P, Babbit J, Drobyski WR, et al. Increasing incidence of adenovirus disease in bone marrow transplant recipients. I Infect Dis. 2004; 169: 775-781.

17. Watcharananan SP, Aver R, Ingsathit A, Malathum K. Adenovirus Disease after Kidney Transplantation: Course of infection and Outcome in Relation to Blood Viral and Immune Recovery. Am J Transplant. 2011; 11: 1308.

18. Bil-Lula I, Ussowicz M, Rybka B, et al. PCR diagnostic and monitoring of adenoviral infections in hematopoetic stem sell transplantation recipients. Arch of Vir. 2010; 155: 2007.

19. Pang XL, Doucette K, LeBlanc B. Monitoring of polyomavirus BK Viruria and Viremia in Renal Allograft Recipients by Use of a QQuantative Real-Time PCR Assay: One-Year Prospective Study. J Clin Microbiol. 2007; 45: 3568.

20. Matłosz B, Durlik M. Interstitial nephritis kidney transplant Polyoma BK virus-induced. Przegląd Epidemiologiczny. 2006; 60: 133-140.

21. Khaled AS. Polyomavirus (BK virus) Nephropathy in Kidney Transplant Patients: A Phatologic Precpective. Yonsei Med J. 2004; 45: 1066.

22. Cimbaluk D, Pitelka L, Kluskens L, Gattuso P. Update on human polyoma virus BK nephropathy. Diag Cytopathol. 2009; 37: 773.

23. Sung H, Choi BH, Jung Y, Kim M. Quantitation of BK Virus DNA for Diagnosis of BK Virus-Associated Nephropathy in Renal Transplantant Recipients. J Korean Med Sci. 2008; 23: 814-818.

24. Giruad G, Priftakis P, Bogdanovic G, et al. BK-viruria and haemorrhagic cystitis are more frequent an allogeneic hematopoietic stem cell transplant patients receiving full conditioning and unrelated HLAmismatched grafts. Bone Marrow Transplant. 2008; 41: 737-742.

25. Giraud G, Bogdanovic G, Priftakis P, Remberg M. The incidence of hemorrhagic cystitis and BK-viruria in allogeneic hematopoietic stem cell recipients according to intensity of conditioning regiment. Hematologica. 2006; 91: 401-404. 